\title{
WORKSHOPS FOR PARAPLEGICS IN JAPAN
}

\author{
By Professor Tamikazu Amako \\ Department of Orthopaedic Surgery, Faculty of Medicine, \\ Kyushu University, Fukuoka, Fapan
}

IT was quite regretful that the re-integration of the paraplegic disabled to the community had not been considered for a long time because of unawareness of the employer of even those paralysed who were quite able and willing to work as a result of vocational training in hospital.

Since the International Stoke Mandeville Games (Paralympics), held in Tokyo in November 1964, Government and private organisations have become interested in rehabilitation and employment of the disabled.

At the decision of the Government, the Ministry of Labour set up a plant in May I965. It is called Nagano plant and is 70 miles west from Tokyo. In this district of the country there are many factories with camera and telecommunicational machinery. The plant shares with these factories in manufacturing the parts. About 50 paraplegics can be admitted there. The conditions of employment for them are as follows:

I. There must be no disability of the upper extremities.

2. They must be efficient in A.D.L.

3. They should not have bedsores or urinary infection.

The levels of the lesion of 56 persons working there vary: I cervical lesion, I3 thoracic, 33 lumbar and 9 cases of cauda equina lesions.

The types of work are assembly of $8-\mathrm{mm}$. cine cameras, assembly of meters and minute motors for cine cameras, drilling and lathing.

The same sort of plant was set up in Beppu in 1966 which admits paraplegics and other disabled. As the paraplegic occupies the majority of the admitted, the facilities of the plant are developed with special consideration to the convenience for them. Twenty-three paraplegics are working there and the rest of the employed are disabled as a result of poliomyelitis, cerebral palsy, amputation, etc. The majority of the paraplegics have lesions of the lower thoracic and lumbar cord and cauda equina.

The types of work at Beppu are bamboo-ware, electronics, etc.

Another plant has recently been built at the foot of Mount Fuji. This plant intends to make parts of radio and television sets. It allows to admit 200 disabled. As it was just completed a couple of months ago, it will soon start working with at present 50 persons. This plant uses chiefly small lathes with special consideration for their placement. The Japanese Government are planning to set up another plant next year.

The common characteristic point of these three plants are special considerations for the building so that the paralysed can use their wheelchairs freely and safely. For example, every corner of the corridor has a mirror to show the traffic from the opposite direction. In addition, wash-stands and lavatories are welldesigned for the paralysed and recreation facilities are also satisfactory. At the plant of Beppu they have a swimming pool, too. 
Regarding their attendance to work, let me show you an example of Negano plant. The slide shows the monthly attendance during the year of 1966. Attendance rate is a little decreased in February and March. But during the rest of the year it shows more than 90 per cent. with average rate of 93 per cent. The rate of attendance can be said to be better than that of some plants with normal healthy workers.

Recurrence of bedsores represents the highest percentage of absenteeism. However, it was less than five among an average of 45 attendances per day. As this plant is situated at the mountainside and in a colder area, frostbite also causes them to leave their post during wintertime from November to March. The cases of frostbite were I.5 persons at daily average. Urinary flare-ups were few; the most frequent in a month were $3 \mathrm{I}$ in total.

Four men left the plants in a year because they changed their jobs.

Fatigue through work was examined in 17 paralysed, in the form of questions and answers in each patient. Nine out of them had fatigue after work, but there was only one who answered that his fatigue lasted during the following day. The remaining eight patients did not feel any fatigue through eight hours' labour. Those who have lesions of higher level of the spinal cord complained more of fatigue. Among I 2 patients who have lesions below the ninth thoracic segment, five complained of fatigue and seven did not. It can be concluded that the level of the lesion of the spinal cord has some relation to fatigue through labour.

The last problem is wages for them. At present their wages vary between I8,000 yen and 25,000 yen per month. This is a little higher than the average juvenile labourer earns in Japan.

$\mathrm{Up}$ to date much money has been spent for the special facilities and welfare for the paraplegic disabled. Therefore, the actual wages are relatively low. However, their total income is not low, since the majority of them have received an accident and invalidity pension. It is the general opinion that employment of these disabled labourers is becoming aware to the public, the more so since the present industrial situation represents a big problem because of lack of labourers in comparison with the surprising development of industry.

Those three plants mentioned above have attracted public attention and we expect such plants will be increased in the near future.

\section{ORGANISATION OF SPINAL UNITS}

By Phillip Harris, F.R.C.S.E., F.R.C.P.E., F.R.C.S.(Glas.), F.R.S.E.

Department of Surgical Neurology, Western General Hospital; the Royal Infirmary, Edinburgh; and the Spinal Unit, Edenhall Hospital, Musselburgh, Scotland

Most new ideas in medicine are not accepted immediately, and the demonstration by a few medical pioneers beginning some 20 years ago, that paraplegic patients could be successfully nursed, treated medically and fully rehabilitated was no exception to this rule. However, the general standard of care, resettlement and 